\title{
Chemical Analysis of Thermal Battery Components
}

\author{
Vernon L. Hammersley and Samuel G. Stuart \\ Naval Surface Warfare Center, 300 Highway 361, \\ Crane, IN 47522-500I
}

\begin{abstract}
A number of methods using analytical chemistry were used to identify and quantify components in a thermal battery. The purpose of the task was to prepare a Material Safety Data sheet (MSDS) so that the battery could be shipped. Wet chemical analysis, $x$-ray diffraction, inductively coupled plasma spectroscopy (ICP) and emission spectroscopy were among the methods used to determine the composition of the various components in the thermal battery.
\end{abstract}

\section{Introduction}

The requirement for a Material safety Data sheet (MSDS) for shipment of hazardous materials necessitates an identification of the composition of items that are to be shipped. Shipment of batteries is no exception. Provision of MSDS's for each individual chemical used to make up the battery without regard to how much of each substance is present may not be acceptable. The only safe way to assure that the MSDS provided is acceptable is to identify every significant chemical component in the battery as well as the quantity of each material. For the thermal battery in question this included the anode, cathode, electrolyte, heat pellets, heat paper, insulator disks, mica disks and steel case. A variety of analytical methods were used to identify and determine quantities of components present.

\section{Teardown}

Components such as the lithium anode and the lithium chloride/potassium chloride electrolyte were sensitive to moisture. The heat pellets were affected by moisture and oxygen. A controlled atmosphere chamber was used for breakdown of the thermal battery. An analytical balance was placed inside the chamber for accurate weighing of samples in preparation for chemical analysis. The chamber was purged with argon and moisture levels were less than two parts per million (ppm). Oxygen levels were between 100 and $400 \mathrm{ppm}$.

The thermal battery was of typical size and easily held manually while a mototool could be manipulated to make a circumferential cut at one end. The internal components were carefully lifted out and the parts were segregated and stored in the chamber until ready for analysis. Each anode assembly, cathode/electrolyte pellet, heat pellet and other individual components were weighed so that the total quantity would be known for the MSDS.

\section{Chemical Analysis}

The anode consisted of a metal grid, thin metal cup and the anode material. The anode material was lithium and iron with traces of aluminum and manganese. The anode material was mixed with water to convert the lithium into the hydroxide which was titrated with standardized hydrochloric acid. This is the standard method used for determining $\mathrm{KOH}$ in battery electrolyte from silver-zinc, nickel-cadmium, nickelhydrogen and other batteries using $\mathrm{KOH}$ electrolyte. The method allows for carbonate and total alkalinity as well as the hydroxide. ${ }^{1}$ The iron was filtered onto absorbent cotton and then determined by reduction with zinc and titration with potassium permanganate. ${ }^{2}$ The details of the iron method are included later in this paper. The amount of iron and lithium in the anode composition was typical for thermal batteries.

The cathode and electrolyte powders, as removed from the thermal battery, were pressed together and could not be separated. The methods of analysis used to sort out the identity and quantity of each component in the cathode/electrolyte wafers were wet chemistry, $x$-ray diffraction and inductively coupled plasma spectroscopy (ICP). X-ray 
diffraction was used to identify the components in the cathode and electrolyte. The cathode was iron disulfide, Fes, which is the mineral iron pyrite. The electrolyte was lithium chloride, potassium chloride and magnesium oxide. One other element of significant quantity was detected by ICP. That element was calcium, presumably present as the oxide. The chloride quantity was determined by dissolving the sample in water, filtering out the insolubles and titrating with silver nitrate solution. The ICP provided the quantities of lithium, potassium, magnesium and calcium. Iithium and potassium were calculated as chlorides using the titration and ICP data. Magnesium and calcium were calculated as oxides. Iron and sulfur in the iron disulfide were both determined by wet chemical methods. A method was found for determining sulfur in pyrite ore. ${ }^{3}$ The iron was recovered as a ferric hydroxide $\left(\mathrm{Fe}(\mathrm{OH})_{3}\right.$ ) precipitate and was dissolved in hydrochloric acid followed by reduction and then titration with potassium permanganate. The sulfur was converted to sulfate by action of bromine and concentrated nitric acid. The sulfate was precipitated with barium chloride solution and weighed as barium sulfate. The insoluble encountered during the pyrite procedure was filtered, dried and analyzed by x-ray diffraction. It was silicon dioxide, a constituent expected in pyrite ore. The cathode composition amounted to $89.4 \% \mathrm{FeS}_{2}$ and 10.6\% $\mathrm{SiO}_{2}$. The electrolyte contained slightly more $\mathrm{KCl}$ than $\mathrm{LiCl}$. Both salts together amounted to $54 \%$ of the total electrolyte composition. The remainder was Mgo with less than $0.5 \% \mathrm{CaO}$.

The heat pellets were composed of potassium perchlorate, $\mathrm{KClO}_{4}$, and iron as determined by $x-r a y$ diffraction. Potassium was determined by ICP and calculated as potassium perchlorate. Iron was determined as previously described by reduction with zinc and titration with potassium permanganate. The reactions involved with the iron reduction and oxidation by permanganate are below.

$$
\begin{aligned}
& 2 \mathrm{Fe}^{+*+}+\mathrm{Zn}=2 \mathrm{Fe}^{+*}+\mathrm{Zn}^{+*} \\
& 5 \mathrm{Fe}^{+*}+\mathrm{MnO}_{4}^{-}+8 \mathrm{H}^{*}=\mathrm{Mn}^{*+}+5 \mathrm{Fe}^{+*}+4 \mathrm{H}_{2} \mathrm{O}
\end{aligned}
$$

Although air oxidation of ferrous iron is very slow, a standard iron powder was reduced and then oxidized according to the permanganate procedure to see what air oxidation may have occurred.
Recovery amounted to $98.59 \%$ iron. All results for iron were adjusted accordingly. The heat pellet composition was consistent with values given in the literature for perchlorate-iron composition.

The heat paper quantity in the thermal battery amounted to one gram. The composition was barium chromate, zirconium and ceramic/asbestos fibers. A typical value for the ceramic and asbestos fibers is $10 \%$ of the total composition. ${ }^{4}$ Zirconium is very slightly soluble and there is no easy method for determining the zirconium in elemental form; therefore it was felt that the 10\% value for the fibers and determination of the chromate could be used to obtain a result for zirconium by difference. The chromate was determined by titration of the iodine liberated with sodium thiosulfate. ${ }^{5}$ A number of reactions take place for this determination. They appear below.

$$
\begin{aligned}
& 2 \mathrm{CrO}_{4}{ }^{--}+2 \mathrm{H}^{+}=\mathrm{Cr}_{2} \mathrm{O}_{7}{ }^{-}+\mathrm{H}_{2} \mathrm{O} \\
& \mathrm{Cr}_{2} \mathrm{O}_{7}^{--}+6 \mathrm{I}^{-}+24 \mathrm{H}^{+}=2 \mathrm{Cr}^{+++}+3 \mathrm{I}_{2}+7 \mathrm{H}_{2} \mathrm{O} \\
& \mathrm{I}_{2}+2 \mathrm{~S}_{2} \mathrm{O}_{3}^{-\cdots}=2 \mathrm{I}^{-}+\mathrm{S}_{4} \mathrm{O}_{6}{ }^{-\cdots}
\end{aligned}
$$

Barium chromate was $59.2 \%$ which left the zirconium at $30.8 \%$.

The insulating and mica disks were identified by $x$-ray diffraction and emission spectroscopy. The mica disk was verified as muscovite, a mineral in the mica group, by $x$-ray diffraction. Emission spectrographic analysis showed aluminum, silicon and magnesium as the major elements. Aluminum and silicon are the main elements in mica. Other base metals can occur in certain minerals of the mica group. X-ray powder cameras were utilized for the identification of the insulating disks. Two possibilities were indicated, kaolinite, $\mathrm{Al}_{2} \mathrm{Si}_{2} \mathrm{O}_{5}(\mathrm{OH})_{4}$, and halloysite, $\mathrm{Al}_{2} \mathrm{Si}_{2} \mathrm{O}_{5}(\mathrm{OH})_{4} 2 \mathrm{H}_{2} \mathrm{O}$. Many of the aluminum and magnesium silicates have similar patterns. Included in that group of compounds is chrysotile, the serpentine mineral known as asbestos. The strong diffraction lines for chrysotile are slightly off from the sample lines. Spectrographic analysis indicated magnesium, calcium, titanium, iron, and boron as additional elements. These elements would be expected in a naturaliy occurring mineral such as kaolinite. The steel case for the thermal 
battery was analyzed by an emission spectrograph using photographic plates. Ten standards, most of which were 300 type stainless steels, were used to compare with the case steel. With the help of the composition tables given in a metals handbook ${ }^{6}$ and the spectrographic results the thermal battery case steel was identified as type 304 stainless steel.

\section{Concluding Remarks}

No one analytical technique can be used to identify the myraid of components in a thermal battery. A combination of instrumental and wet chemical techniques was required for the completion of this task. Although wet chemical methods of analysis are not considered as "glamorous" as instrumental methods they are a necessary part of an analytical task as complex as characterization of a thermal battery. Wet chemistry is necessary for sample preparation prior to analysis by an instrument such as the ICP. Major elements cannot be accurately determined oftentimes except by the classical wet chemistry techniques. These methods worked very well for the quantitative analysis of iron and sulfur. The methods for quantitative determination of elements such as lithium and potassium are long and laborious by wet chemistry so instrumental techniques such as ICP are required. The determination of lithium and potassium by ICP supplemented by chloride analysis by titration gave the quantities of LiCl and $\mathrm{KCl}$ in the electrolyte.

\section{Acknowledgments}

Thanks are due to $\mathrm{Mr}$. Samuel stuart, the coauthor, for valuable input in what to expect for composition of the various components. Appreciation is extended to Mr. Bob Hardwick for his patience in the teardown process of the thermal battery in the dry box. Thanks go to Ms. Teresa Myers for preparation of the presentation slides. Thanks to Mr. Bradley secrest for allowing the paper to be submitted and presented. Gratitude is extended to Dr. Harlan Lewis for providing a time period in the lab relatively free of interruptions in which to complete the task of characterizing the thermal battery.

\section{REFERENCES}

1. Analytical Chemistry, Vol II, Quantitative, Treadwell and Hall, John Wiley \& Sons, New York, 1942, pp 498499 .

2. Applied Inorganic Analysis, 2nd edition, Hillibrand, Lundell, Bright and Hoffman, John Wiley \& Sons, New York, 1953, p 395.

3. Analytical Chemistry, Vol II, Quantitative, Treadwell and Hall, John Wiley \& Sons, New York, 1942, p 315. 4. Private communication, Clinton Winchester, NSWC Carderock, MD.

5. Scott's standard Methods of Chemical Analysis, 6 th edition,

Vol I, N.H. Furman editor, D. Van Nostrand Co., New York, 1962, p 360 .

6. Metals Handbook, 9th edition, Vol 3, Properties and selection: stainless Steels, Tool Materials and Special Purpose Metals, American Society for Metals, 1980, p 9. 